\title{
The effect of denture restoration and dental implant restoration in the treatment of dentition defect: a systematic review and meta- analysis
}

\author{
Kaihui Peng ${ }^{1,2 \#}$, Yueping Zhou ${ }^{3,4 \#}$, Yinhua Dai ${ }^{1,2}$, Qianxia Wang ${ }^{1,2}$, Yujiao Hu ${ }^{1,2}$, Qun Dai ${ }^{1,2}$ \\ ${ }^{1}$ Department of General Emergency, The Affiliated Stomatological Hospital of Nanchang University, Nanchang, China; ${ }^{2}$ The Key Laboratory of \\ Oral Biomedicine, Nanchang, China; ${ }^{3}$ School of Public Health, Nanchang University, Nanchang, China; Jiangxi Key Laboratory of Preventive \\ Medicine, Nanchang, China \\ Contributions: (I) Conception and design: K Peng, Q Dai; (II) Administrative support: Q Dai; (III) Provision of study materials or patients: K Peng, Q \\ Dai; (IV) Collection and assembly of data: Y Zhou, Y Dai; (V) Data analysis and interpretation: K Peng, Y Zhou, Q Dai; (VI) Manuscript writing: All \\ authors; (VII) Final approval of manuscript: All authors. \\ "These authors contributed equally to this work. \\ Correspondence to: Qun Dai. The Affiliated Stomatological Hospital of Nanchang University, No. 49 Fuzhou Road, Donghu District, Nanchang \\ 330000, China. Email: 512968304@qq.com.
}

Backgroundk Dentition defect is a common symptom in clinical dental patients. This study compared the clinical effects of denture restoration and dental implant restoration in the treatment of dentition defects through meta-analysis.

Methods: Data retrieval was conducted through the PubMed, Web of Science, Embase, CNKI, and Wanfang databases. A total of 479 related literatures published in English or Chinese from 2013 to 2020 were included. Literature screening, data extraction and comprehensive evaluation, and analysis by metaanalysis was performed by 3 authors.

Results: A total of 17 studies and 1,459 patients were included. Among the 17 studies, the effective rate of treatment between the two groups was compared and the experimental group rate was significantly higher than that of the control group [odds ratio $(\mathrm{OR})=6.149$, 95\% confidence interval (CI): 4.103-9.215, $\mathrm{P}<0.001$ ]; the mastication function score was compared, and was higher in the experimental group than in the control group [standardized mean difference $(\mathrm{SMD})=1.632,95 \% \mathrm{CI}: 1.039-2.224, \mathrm{P}<0.001$ ]; the retention function score was compared, and was higher in the experimental group than in the control group (SMD $=1.775$, 95\% CI: 1.095-2.455), $\mathrm{P}<0.001)$; the aesthetics score was also compared, and was higher in the experimental group than in the control group ( $\mathrm{SMD}=1.300,95 \% \mathrm{CI}: 0.499-2.100, \mathrm{P}=0.001)$. Among 17 studies, 15 compared the comfort score, which was higher in the experimental group than in the control group (SMD $=1.357,95 \%$ CI: 0.455-2.258, P=0.003).

Conclusions: Compared with denture restoration, dental implant restoration is more effective in the treatment of dentition defect with a higher comprehensive score of functional restoration.

Keywords: Dental implant restoration; denture restoration; dentition defect; clinical effect; meta-analysis

Submitted Jan 21, 2021. Accepted for publication Mar 23, 2021.

doi: $10.21037 /$ apm-21-421

View this article at: http://dx.doi.org/10.21037/apm-21-421 


\section{Introduction}

Dentition defect is a common symptom in clinical dental patients, which is not conducive to occlusion, chewing, and eating. It may cause oral mucosal issues, deterioration of jaw joints, facial muscles, and nerves, and even the abnormal wear of adjacent teeth, thus affecting overall oral health (1). It was shown in a national survey that only $13.8 \%$ of adults had intact dentition, with a prevalence of $84.4 \%$ for dentition defects and $1.8 \%$ for edentulism. However, the rate of dentition defect restoration accounts for only $41.6 \%$ of the national adult population (2). With the continuous development of clinical medical treatment and prosthodontic techniques, there are increasing methods used to treat dentition defects, including denture restoration and implant denture restoration. Denture restoration use artificial prosthesis to repair missing dentition, which can be divided into fixed denture, overdentures and removable denture. Implant denture uses the principle of osseointegration to implant the implant made of artificial materials into the bone tissue, and connect the upper dental prosthesis through a special way, so as to carry out dentition repair $(3,4)$. Although many studies have reported that the clinical effect of dental implant restoration in the treatment of dentition defect is better compared with denture restoration (5), its efficacy and degree of functional restoration still remain to be systematically and comprehensively evaluated. Thus, this study explored the clinical effect of denture restoration and dental implant restoration in patients with dentition defect in order to comprehensively compare the clinical efficacy of these two methods.

We present the following article in accordance with the PRISMA reporting checklist (available at http://dx.doi. org/10.21037/apm-21-421).

\section{Methods}

\section{Literature retrieval methods}

Database search words were set as follows: "denture restoration, dental implant restoration, dentition defect", ("fixed prosthesis" or "implant-supported denture") or ("dental implant restoration" or "dental implant repair"), and ("dentition defect" or "dentition loss"). Searches were conducted between 2013 and 2020. The initial search was performed in foreign language databases such as PubMed, Web of Science and Embase, and Chinese databases such as CNKI and Wanfang. References, conference papers, abstract articles, and other possible selected documents of relevant literature were located during the second round search.

\section{Inclusion and exclusion criteria}

Based on PICOS principle-participants, interventions, comparisons, outcomes, and study design, the following criteria were adopted: (I) participants (P): patients who were definitely diagnosed with dentition defect (II) interventions (C, I) were randomly divided into the experimental group and control groups. Participants in the experimental group were treated with dental implant restoration, while those in the control group were treated with denture restoration. The effect of treatment of dentition defect was analyzed. (III) observation index (O): (i) curative effect: (a) successful repair, pronunciation and masticatory functions returned to normal, and no loose denture phenomenon showed a marked effect; (b) the restoration was basically successful. Compared with before treatment, the pronunciation and chewing functions were significantly improved, and slight loosening of the denture was effective. c. Imperfect repair. Compared with before treatment, no improvement in pronunciation and chewing functions indicated no effect. (ii) Comparison of comprehensive scores of functional rehabilitation between the two groups: chewing function; fixed function; beauty; comfort score. (IV) Study design (S): all clinical studies comparing the effect of denture restoration and dental implant restoration in the treatment of dentition defect.

Exclusion criteria were as follows: (I) not a case-control study for the treatment of dentition defects; (II) case reports, letters, review articles; (III) duplicate report studies; (IV) studies with unclear conclusions such as insufficient data and design defects.

\section{Literature quality evaluation and data extraction}

The risk of bias in each identified study was assessed by using the Cochrane Collaboration tool. The tool considered 6 different domains: (I) generation of random sequences (selection bias); (II) allocation concealment (selection deviation); (III) participant and personnel blindness (performance bias); (IV) blind assessment of results (detection bias); (V) incomplete outcome data (attrition bias); and (VI) selective reporting (reporting deviation).

Independent literature assessment and data extraction was performed separately by two investigators for the study, 


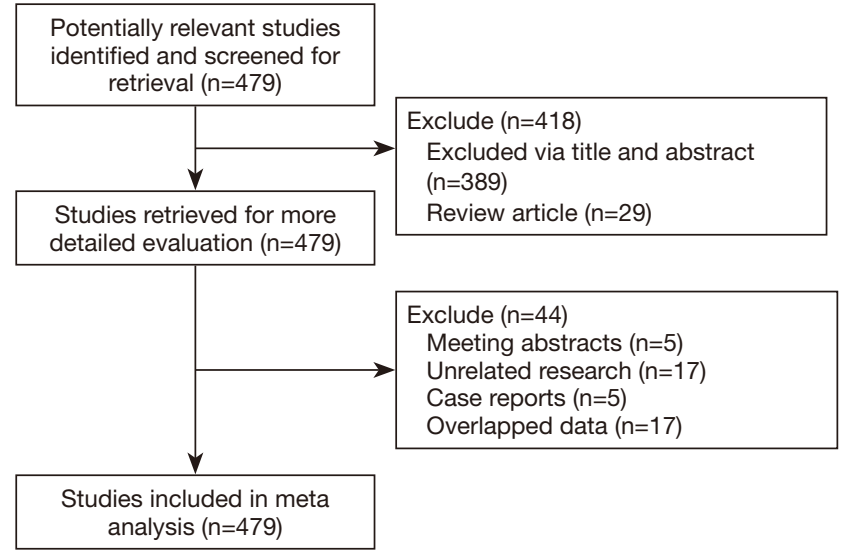

Figure 1 Flow chart of included literatures. n, numbers of literatures.

and any disagreements in data extraction were discussed and negotiated with a third researcher for resolution.

\section{Statistical analysis}

Statistical analysis was conducted using the software Stata (16.0 StataCorp LLC, College Station, TX, USA). Heterogeneity of included studies was assessed using Cochran's $\mathrm{Q}$ test and $\mathrm{I}^{2}$ statistics. When $\mathrm{P}<0.05$ and $\mathrm{I}^{2}>50 \%$, the random-effects model was used for meta-analysis; otherwise, the fixed-effects model was used. The results of categorical variable data were reported as odds ratio $(\mathrm{OR})$ and $95 \%$ confidence interval (CI), while the results of numerical variable data were reported as standardized mean difference (SMD) and its $95 \%$ CI. A P value $<0.05$ was considered statistically significant. Sensitivity analysis was used to evaluate the degree of robustness and credibility of the results of the meta-analysis. Funnel plots were applied to analyze the results for the presence of publication bias.

\section{Results}

\section{Literature retrieval results and characteristics}

Preliminarily, 479 articles were retrieved, and 61 were included after 418 unqualified articles were removed according to the title, abstract, and full text. Given the bias of some studies as well as the requirements of research methods, a final 17 studies met the selection criteria (6-22) (Figure 1). All 17 articles were Chinese papers, with a cumulative number of 1,459 cases, including 728 cases in the dental implant restorative group (experimental group) and 731 cases in the denture restorative group (control group). Table 1 summarizes the basic characteristics of all included articles.

\section{Meta-analysis results and results}

A total of 17 included studies compared the effectiveness of repair between the two groups. The pooled data showed great heterogeneity among studies $\left(\mathrm{I}^{2}=0.00 \%, \mathrm{P}=0.999\right)$, so the random-effects model was used. Meta-analysis showed that the effective rate of treatment in the experimental group was significantly higher than that in the control group (OR =6.15, 95\% CI: 4.10-9.22, $\mathrm{P}<0.001)$ (Figure 2).

A total of 17 studies further compared the masticatory function scores, and the combined data showed that each study had great heterogeneity $\left(\mathrm{I}^{2}=95.8 \%, \mathrm{P}<0.001\right)$, so the random-effects model was used, and the scores of the experimental group were significantly higher than those of the control group (SMD $=1.63,95 \%$ CI: 1.04-2.22, $\mathrm{P}<0.001$ ) (Figure $3 A$ ).

The 17 studies compared the scores of retention function. The data showed great heterogeneity among the studies after combination $\left(\mathrm{I}^{2}=96.6 \%, \mathrm{P}<0.001\right)$. Therefore, the randomeffects model was used. The score of the experimental group was higher than that of the control group (SMD $=1.77,95 \%$ CI: $1.10-2.45, \mathrm{P}<0.001$ ) (Figure $3 B$ ).

The aesthetics scores of 17 studies were compared. The combined data showed great heterogeneity among the studies $\left(\mathrm{I}^{2}=97.5 \%, \mathrm{P}<0.001\right)$. Therefore, the random-effects model was used. The scores of the experimental group were higher than those of the control group (SMD $=1.30,95 \%$ CI: 0.50-2.10, $\mathrm{P}=0.001$ ) (Figure 3C).

A total of 15 studies compared the comfort score. The data showed great heterogeneity among studies after combination $\left(\mathrm{I}^{2}=97.7 \%, \mathrm{P}<0.001\right)$. Therefore, the randomeffects model was used. The experimental group score was higher than that of the control group (SMD $=1.36,95 \% \mathrm{CI}$ : 0.46-2.26, $\mathrm{P}=0.003$ ) (Figure 3D).

\section{Sensitivity analyses}

The results of sensitivity analysis showed that after changing the inclusion criteria, excluding low-quality studies, removing the maximum weight, and removing the minimum weight literatures, the combined results of the effective rate of the excluded literatures did not change much from the original combined results; therefore, it could indicate that the sensitivity was low (Figure 4). In addition, 


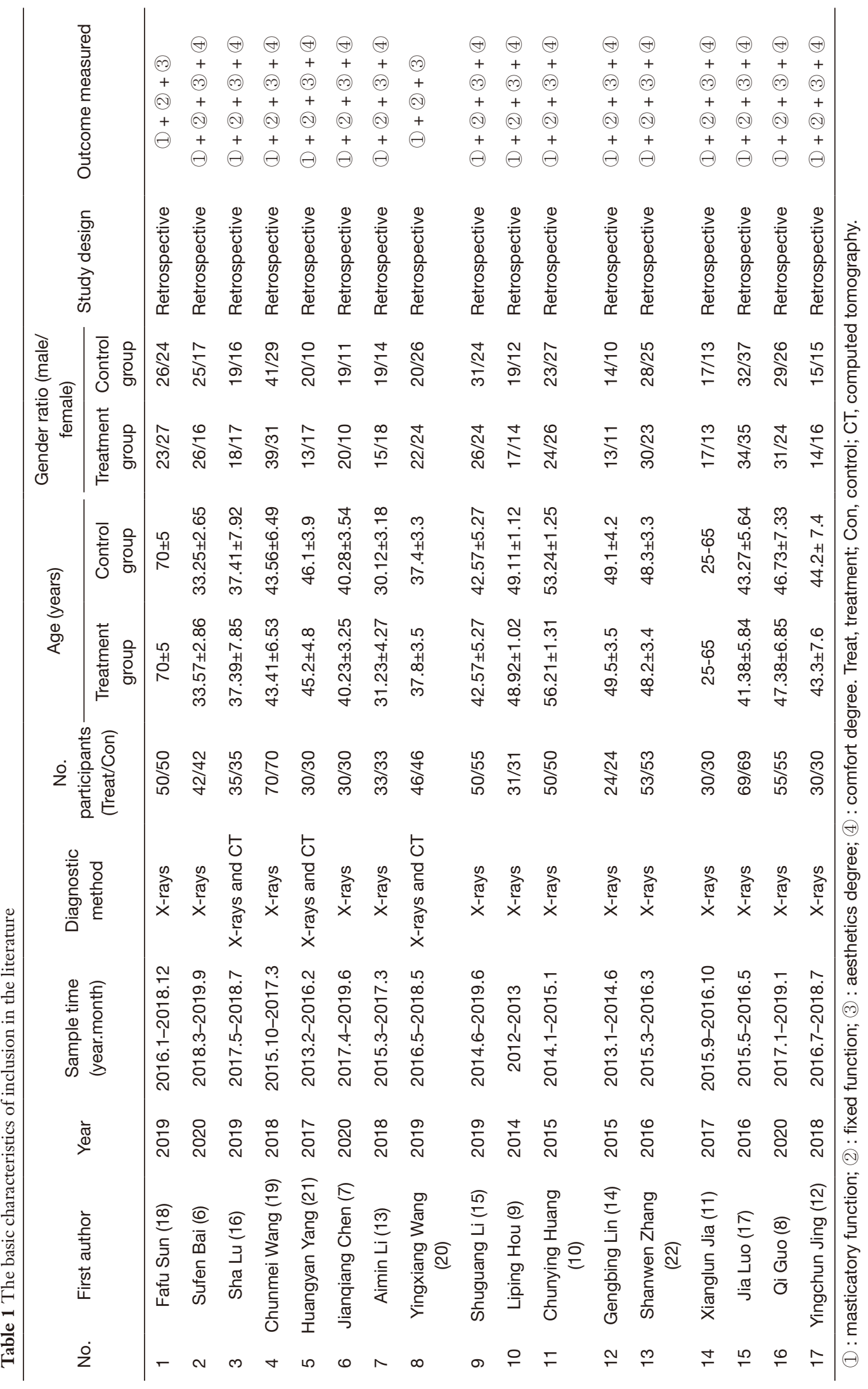




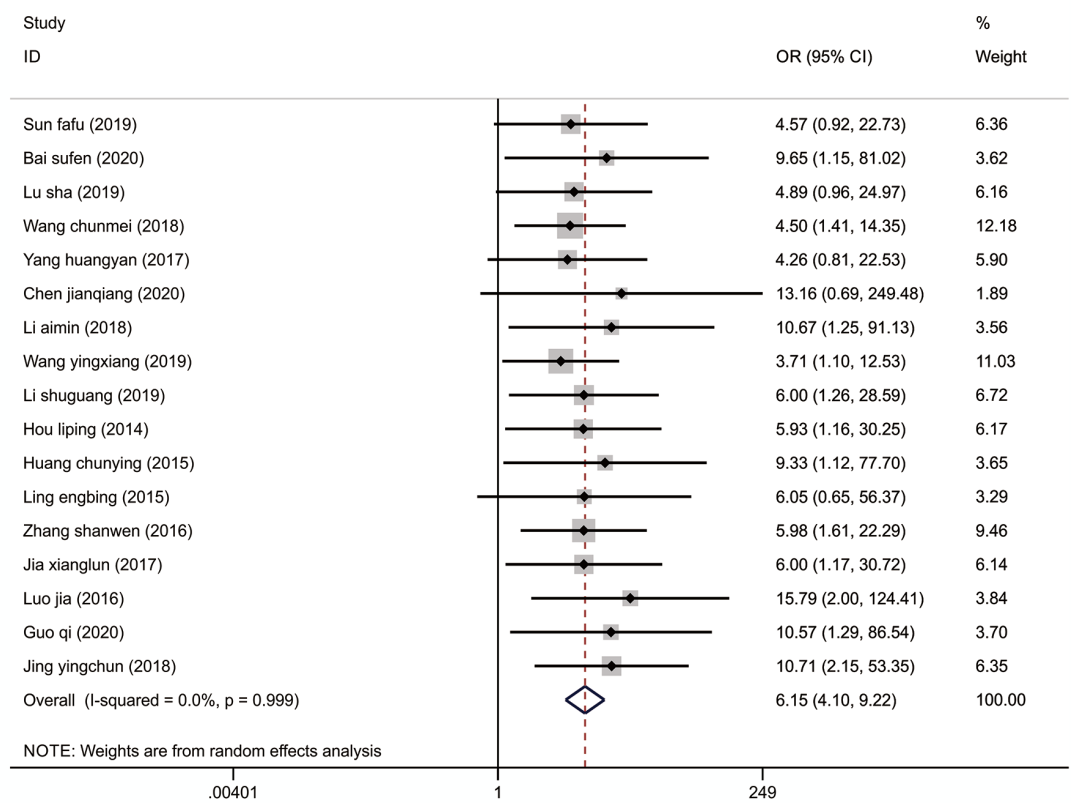

Figure 2 Forest map of the effective rate of dentition defect in patients with dental implant restoration and denture restoration. OR, odds ratio; $\mathrm{CI}$, confidence interval.

the combined results of masticatory function score, retention function score, aesthetics, and comfort of each study did not change much from the original combined results (Figure $5 A, B, C, D$ ). These results confirmed that the results of this meta-analysis were robust and credible.

\section{Publication bias}

Funnel plots were used to detect whether there was publication bias in the selected articles. The results of Figure 6 showed that with the effective rate se $(\log \mathrm{OR})$ and $\log \mathrm{OR}$, the scatter diagram basically showed leftright symmetry around the symmetry axis, confirming that there was no significant publication bias in various studies. Subsequently, we made a funnel plot of se (SMD) and SMD of masticatory function score, retention function score, aesthetics, and comfort (Figure $7 A, B, C, D$ ). It can be seen that the scatter plot basically presents left-right symmetry about the symmetry axis, indicating that various studies did not show the main publication bias.

\section{Discussion}

Dentition defects are very common in clinical dental cases, mainly involving partial or complete loss of teeth, resulting in incomplete dentition, and affecting the normal function of teeth and tooth aesthetics. Dental defects may occur at any age. Among them, the elderly population has the highest prevalence, mostly due to alveolar bone osteoporosis and periodontal and dental degeneration, which leads to poor tooth fixation and loosening and loss, resulting in dentition defects (23). The cause of loss in other populations may be accidental collisions during strenuous exercise, resulting in tooth impingement and loss (7). In recent years, with the continuous development of medical technology, prosthodontic technology has been gradually improved. There are a variety of restorative methods for dentition defects in clinical practice, and denture restorations and dental implant restorations are common. Dentures can be further divided into removable and fixed types. This analysis was mainly about fixed denture restorations, which involve the use of adhesives or fixtures that are fixed with implants or abutments adjacent to the missing tooth, and act as substitutes for the normal physiological function of the missing tooth (24). Dental implant restoration is a new and effective way to repair dentition defects by drilling holes with appropriate depth and angle in the dentition defect and placing implants in it. Advanced technology, equipment, and appropriate implants are applied during the operation (25). 
A

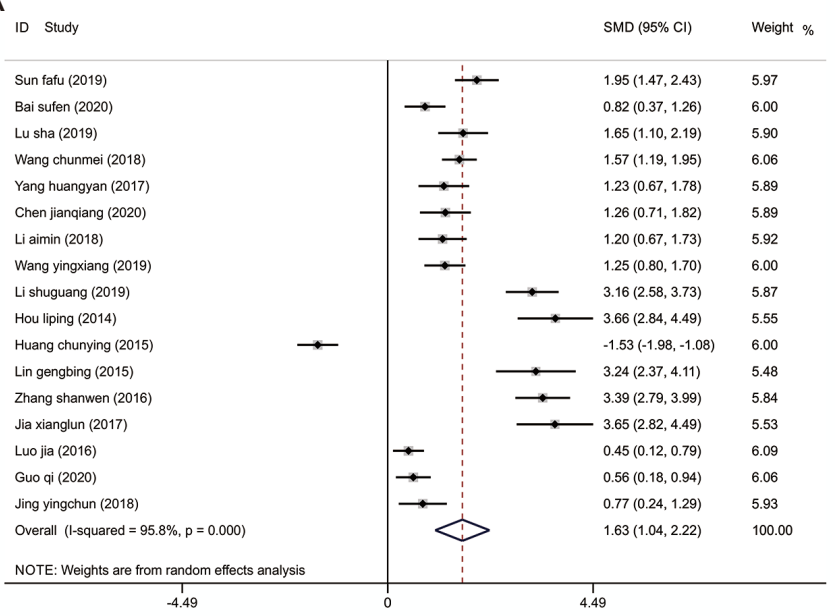

C

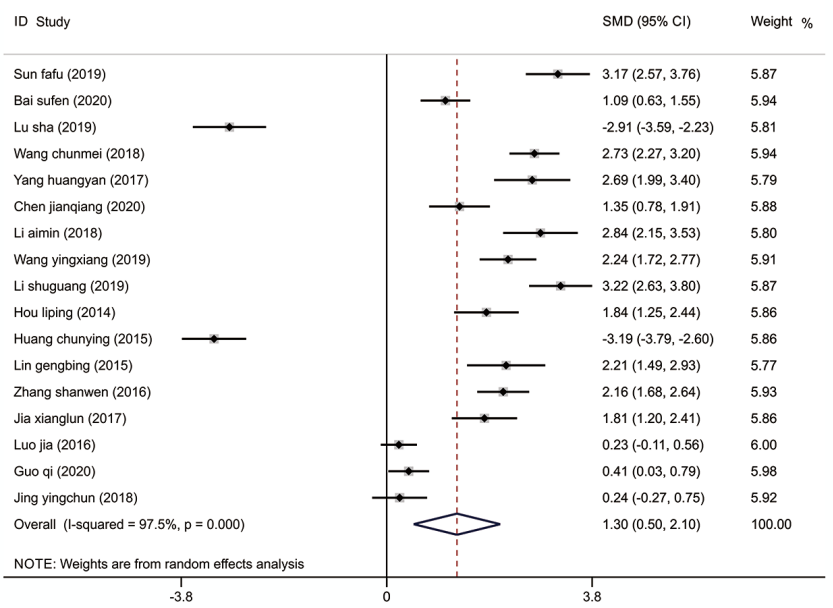

B

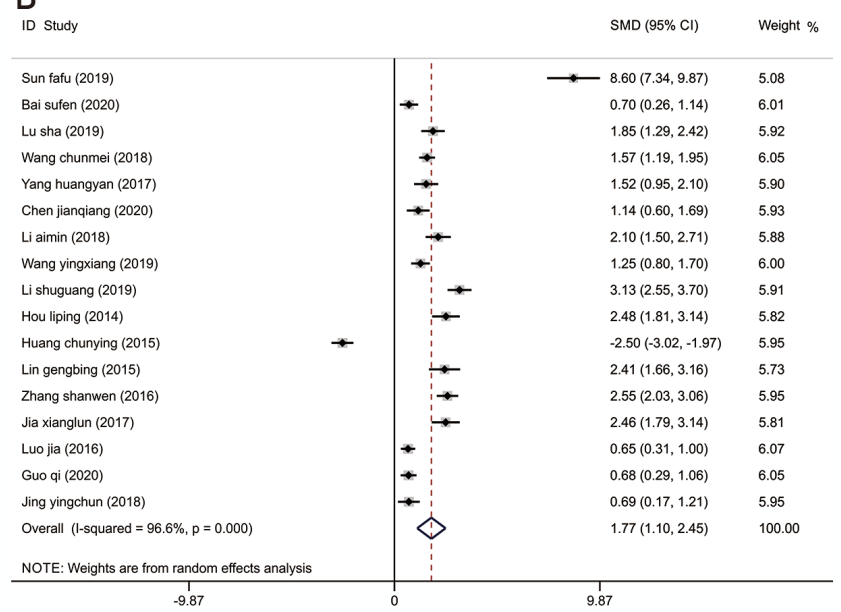

D

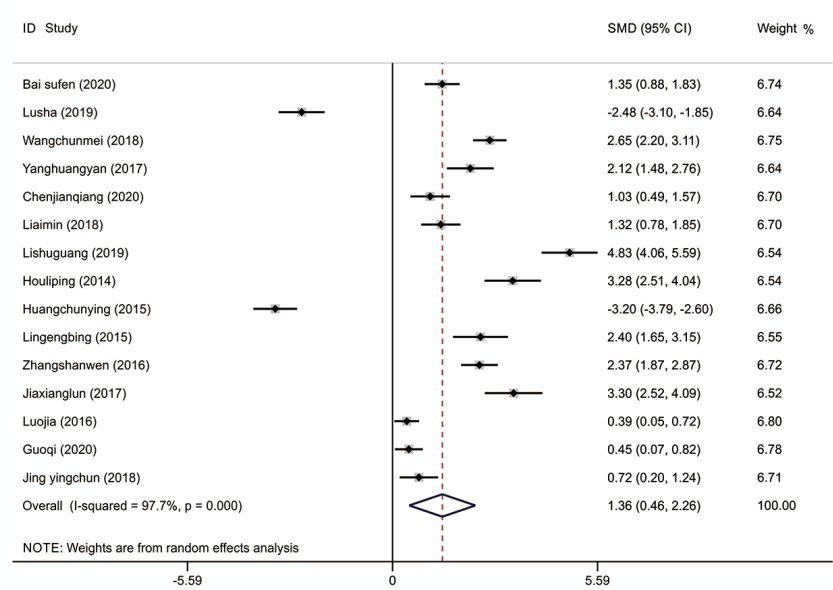

Figure 3 Forest map of comprehensive functional restoration scores of participants in the two groups. (A) Masticatory function score; (B) retention function score; (C) aesthetics; (D) comfort. OR, odds ratio; CI, confidence interval.

A total of 17 studies included in this paper compared the effective rate of restoration between the two groups, and the results revealed that the treatment effective rate of dental implant restoration was significantly higher than that of the denture restoration control (OR $=6.149,95 \%$ CI: 4.103-9.215, $\mathrm{P}<0.001)$. The clinical effective rate is mainly judged by whether masticatory function is normal after treatment, whether the prosthesis is loose and fractured, and whether the abutment teeth are painful (14). Related studies have mentioned that possible reasons may be that the fixed denture does not need to be worn and has good fixation, but the simultaneous fixed denture restoration requires abrasion of the surrounding tooth tissue, so it is suitable for patients with a small number of tooth defects (26). At the same time, fixed dentures require a high health status of the surrounding teeth and are not suitable for the characteristics of poor abutment stability and alveolar ridge morphology in elderly patients (27), which are prone to the risk of pulp exposure and postoperative complications of endodontic teeth (28), affecting the clinical efficacy of tooth restoration. Implant restoration can reduce the damaging effect on periodontal tissues, reduce the marginal bone resorption of implants, and effectively improve the clinical success and retention rates (29); thus, the treatment efficiency of the implant restoration group was significantly higher than that of the 


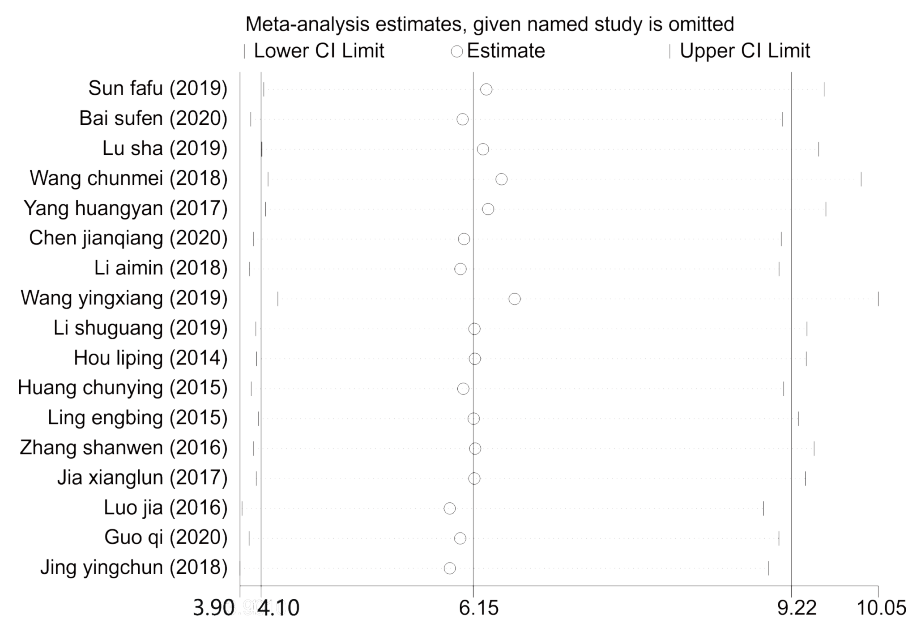

Figure 4 Sensitivity analysis plot of response rate. CI, confidence interval.

A Meta-analysis estimates, given named study is omitted

Sun fafu (2019) Bai sufen (2020) Lu sha (2019) Wang chunmei (2018) Yang huangyan (2017)

Chen jianqiang (2020) Li aimin (2018)

Wang yingxiang (2019)

Li shuguang (2019)

Hou liping (2014)

Huang chunying (2015)

Lin gengbing (2015)

Zhang shanwen (2016)

Jia xianglun (2017)

Luo jia (2016)

Guo qi (2020)

Jing yingchun (2018)

Lower CI Limit Estimate Upper CI Limit

0.921 .04

1.63

Meta-analysis estimates, given named study is omitted

C

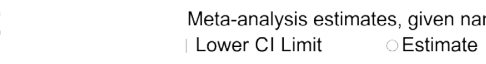

Sun fafu (2019)

Bai sufen (2020)

Lu sha (2019)

Wang chunmei (2018)

Yang huangyan (2017)

Chen jianqiang (2020)

Li aimin (2018)

Wang yingxiang (2019)

Li shuguang (2019)

Hou liping (2014)

Huang chunying (2015)

Lin gengbing (2015)

Zhang shanwen (2016)

Jia xianglun (2017)

Luo jia (2016)

Guo qi (2020)

Jing yingchun (2018)

Lower CI Limit

Upper Cl Limit

2.222 .34

$$
0.370 .50
$$

B

Meta-analysis estimates, given named study is omitted

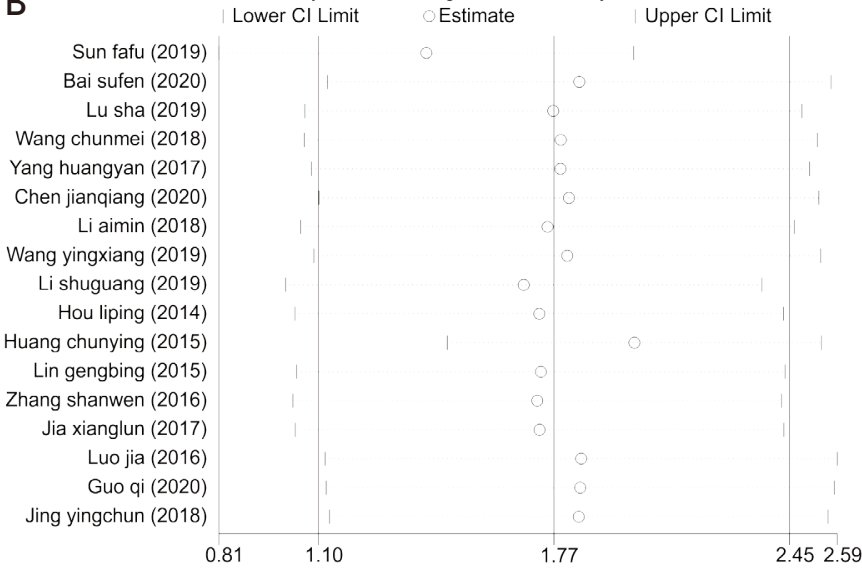

D

Meta-analysis estimates, given named study is omitted

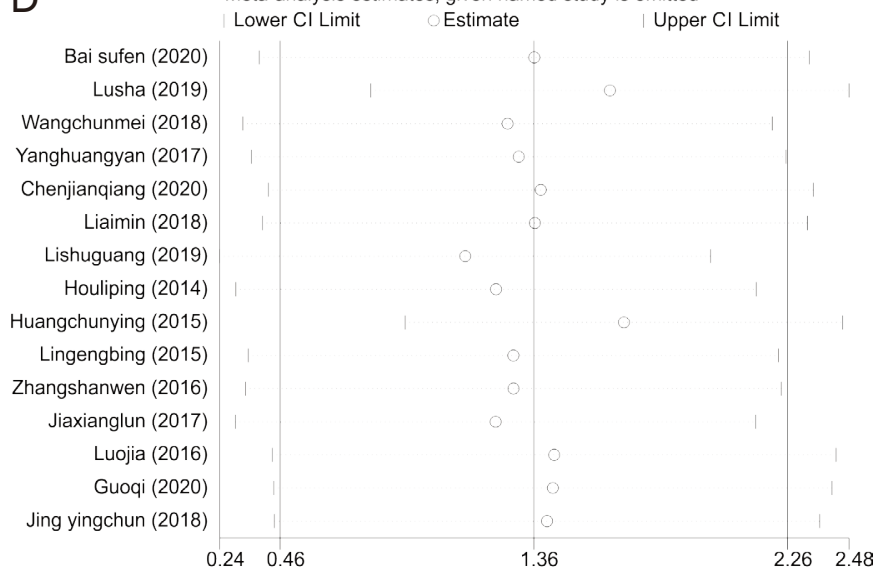

Figure 5 Sensitivity analysis of functional restoration composite score. (A) Masticatory function score; (B) retention function score; (C) aesthetics; (D) comfort. CI, confidence interval. 
control group.

Moreover, this paper further evaluated the comprehensive results of functional restoration in the two groups of patients, a total of 17 studies compared the masticatory function score, retention function, and 3 aesthetics indicators, 15 studies compared the comfort score, and all found that the score in the implant restoration group was significantly higher than that of the denture

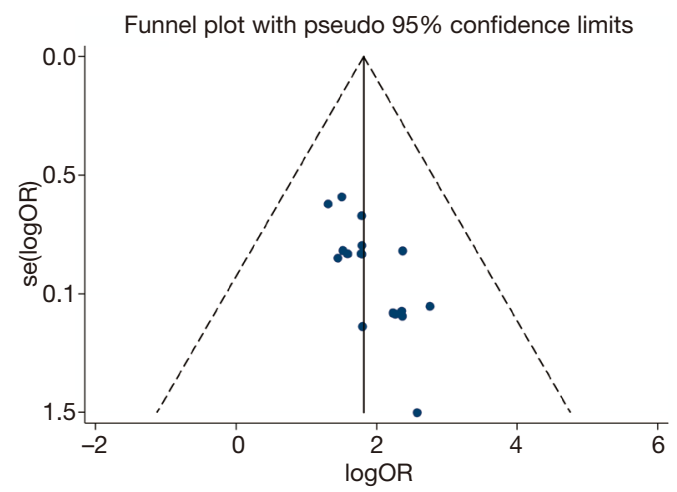

Figure 6 Funnel plot of effective rate. OR, odds ratio.
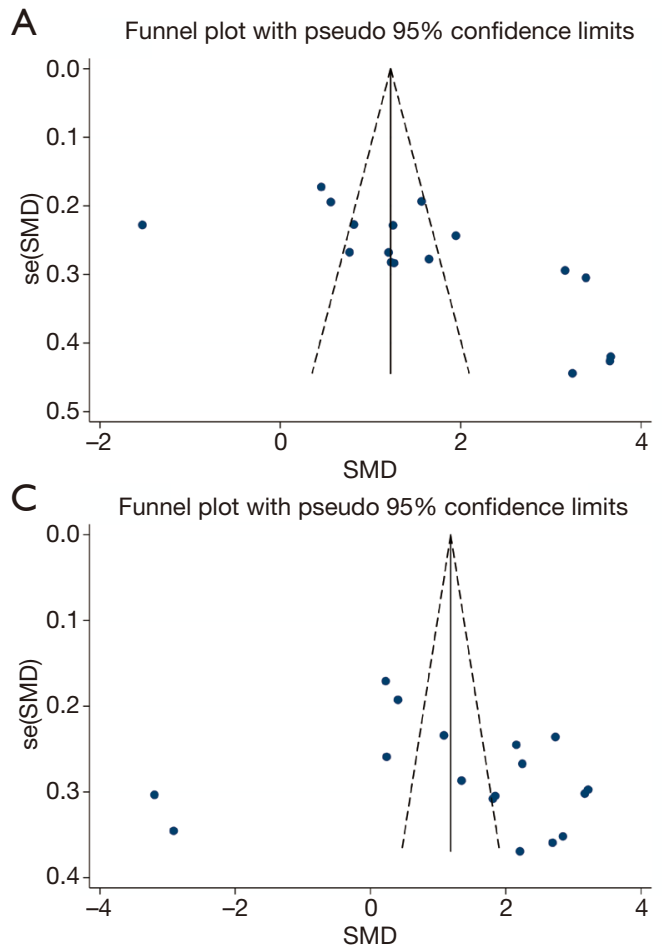

restoration group. Fixed dentures require fixing with the help of periodontal reserve forces (30), which may affect masticatory function and the function of restorations and abutments. Fixed dentures rely on the health status of surrounding teeth and can play a good retention function after restoration in the case of adjacent teeth and periodontal health, so the retention effect is poorer than that of implant restorations. Compared with fixed dentures, the application of dental implants in prosthodontics can greatly improve aesthetics and is easier for patients to accept and recognize (30). The analysis is mainly because the raw material used for implant dentures is pure titanium metal, which is both corrosion resistant and biocompatible, and the crowns are made using simulation technology with high aesthetic attention. Compared with dentures, dental implants are less damaging to both teeth because they do not use bases and clasps, have better comfort, have less foreign body sensation in patients, and have no effect on pronunciation or swallowing (31). Fixed dentures place more implants supporting dentures during treatment, which can cause a foreign body sensation in the oral cavity and affect patient comfort (32).
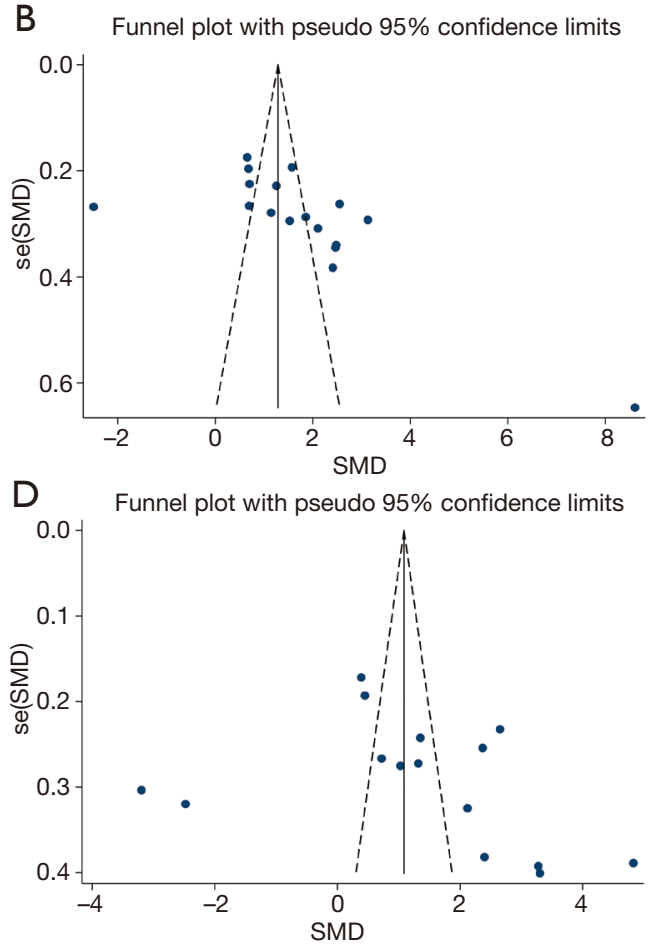

Figure 7 Funnel plot of comprehensive patient scores of functional restoration. (A) Masticatory function score; (B) retention function score; (C) aesthetics; (D) comfort. SMD, standardized mean difference 
This meta-analysis had some limitations. First, most of the included studies were Chinese literatures, and the study sample size was small, which may have some selection bias and affect the extrapolation of a conclusion. Second, the study did not employ a blind method and allocation concealment scheme to control information bias, which may affect the internal authenticity of the results.

In summary, compared with denture restoration, dental implant restoration is more effective in the treatment of dentition defects, with a comprehensive score. In addition, it should be noted that before treatment, it is necessary to conduct a comprehensive examination and evaluation of dental condition, determine the number, location, and specific angle of dentition defects and other issues, select the appropriate, scientific, and rigorous restorative plan according to the specific circumstances of the oral cavity, strictly implement aseptic operation during surgery, and standardize the operating process. At the end of implantation, corresponding nursing should be performed to maintain oral health.

\section{Acknowledgments}

Funding: None.

\section{Footnote}

Reporting Checklist: The authors have completed the PRISMA reporting checklist. Available at http://dx.doi. org/10.21037/apm-21-421

Conflicts of Interest: All authors have completed the ICMJE uniform disclosure form (available at http://dx.doi. org/10.21037/apm-21-421). The authors have no conflicts of interest to declare.

Ethical Statement: The authors are accountable for all aspects of the work in ensuring that questions related to the accuracy or integrity of any part of the work are appropriately investigated and resolved.

Open Access Statement: This is an Open Access article distributed in accordance with the Creative Commons Attribution-NonCommercial-NoDerivs 4.0 International License (CC BY-NC-ND 4.0), which permits the noncommercial replication and distribution of the article with the strict proviso that no changes or edits are made and the original work is properly cited (including links to both the formal publication through the relevant DOI and the license). See: https://creativecommons.org/licenses/by-nc-nd/4.0/.

\section{References}

1. Beck JD, Youngblood M Jr, Atkinson JC, et al. The prevalence of caries and tooth loss among participants in the Hispanic Community Health Study/Study of Latinos. J Am Dent Assoc 2014;145:531-40.

2. Guo J, Ban J, Wang X, et al. A national sample survey of tooth loss and denture restoration in Chinese adults. Proceedings of the 18th Annual Academic Conference of Oral Preventive Medicine; 2018 Oct 26-27; Xi'an, China. Chinese Society of Stomatology, 2018.

3. Li Y, Liang Q, Zhao W, et al. Aesthetic observation and curative effect evaluation of oral implant in repairing dentition defect. China Foreign Medical Treatment 2015;34:33-4, 37.

4. Yelick PC, Sharpe PT. Tooth Bioengineering and Regenerative Dentistry. J Dent Res 2019;98:1173-82.

5. Kutkut A, Bertoli E, Frazer R, et al. A systematic review of studies comparing conventional complete denture and implant retained overdenture. J Prosthodont Res 2018;62:1-9.

6. Bai S. To compare the clinical efficacy of oral implant and conventional fixed denture in the treatment of dentition defects. Psychology Monthly 2020;15:214.

7. Chen J. Evaluation of the effect of dental implant restoration and removable denture restoration in the treatment of dentition defects. Forum on Primary Care Medicine 2020;24:2414-5.

8. Guo Q. Analysis of the effect of dental implant in the treatment of 55 cases of dentition defect. Henan Journal of Surgery, 2020.

9. Hou L and Li E. Clinical effect of oral implant in repairing dentition defect. Chinese Journal of Oral Implantology 2014;19:190-1.

10. Huang C. Clinical efficacy analysis of oral implant in repairing dentition defect. World Latest Medicine Information: Electronic Edition, 2015.

11. Jia X. Clinical effect of oral implant in repairing dentition defect. World Latest Medicine Information 2017;(61):61.

12. Jing Y. Effect of denture restoration and oral implant restoration on dentition defect. Journal of General Stomatology 2018;5:58, 60 .

13. Li A. Comparison of the effect of dental implant restoration and traditional fixed denture restoration in the treatment of dentition defects. Journal of General 
Stomatology 2018;5:93-4.

14. Lin G. Clinical efficacy analysis of oral implant in repairing dentition defect. Modern Diagnostics \& Therapeutics 2015;26:3290-1.

15. Li S, Wang $\mathrm{N}$ and Li X. Effect of dental implant restoration in patients with edentulism. Zhejiang Journal of Trauma Surgery 2019;024:1113-4.

16. Lu S. Dental implant restoration and traditional fixed denture restoration in the treatment of dentition defects. Journal of General Stomatology 2019;6:88-91.

17. Luo J, Yang Y. Aesthetic value and clinical effect of dental implant in repairing dentition defect. Hebei Medical Journal 2016;22:1971-3.

18. Sun F, Li M, Wang Y, et al. Clinical effects of oral implant overdenture restorations compared with complete denture restorations for edentulism. Journal of General Stomatology 2019;6:40-2.

19. Wang C. Clinical comparison of dental implant restoration and traditional fixed denture restoration in the treatment of dentition defects. Grassroots Medical Forum 2018;22:2783-84.

20. Wang Y. Clinical effect of dental implant restoration and fixed denture restoration in the treatment of dentition defect. China Modern Medicine 2019;026:132-4.

21. Yang Y, Zhang X, Luo J, et al. Comparison of the effects of oral implant restorations and traditional fixed denture restorations in the treatment of dentition defects. China Medical Science 2017;7:199-201.

22. Zhang S, Liu X, Yu P, et al. Clinical efficacy of oral implants in the repair of dentition defects. Journal of General Stomatology 2016;3:35-7.

23. Wei Y, Liu Y, Bai Q. Effect of oral implant restoration and conventional restoration on the therapeutic effect

Cite this article as: Peng K, Zhou Y, Dai Y, Wang Q, Hu Y, Dai Q. The effect of denture restoration and dental implant restoration in the treatment of dentition defect: a systematic review and meta-analysis. Ann Palliat Med 2021;10(3):32673276. doi: 10.21037/apm-21-421 and prognosis of patients with dentition defect. Journal of Clinical and Experimental Medicine 2017;16:2171-3.

24. Xie F. Application of removable denture and fixed denture in the repair of periodontal disease with dentition defect. Chinese Journal of Otorhinolaryngology of Integrated Traditional and Western Medicine 2016;24:453-6, 461.

25. Huang F. Clinical effect of repairing dentition defect with dental implant. China Medical Innovation, 2019.

26. Teng J. Effect of oral implant restoration on implant quality in patients with periodontitis dentition defect. Chinese Journal of Clinical Drugs 2019;19:2045-7.

27. Li Y. Elderly patients denture restoration design principles. Chinese Journal of Geriatric Stomatology 2013;(1):63.

28. Xing F, Hao N, Li K, et al. Effect of fiber-reinforced composite resin adhesive bridge on survival rate and curative effect of premolar loss. Shanxi Medical Journal 2016;45:800-2.

29. Wang L, Guo X, Dong Y, et al. Short-term clinical efficacy of Trausim implant for dentition defects. Oral Research 2018;34:675-8.

30. Chen Y. Comparison of implant denture and fixed denture in prosthodontics. Chinese Journal of Oral Implantology 2018;23:139-41.

31. Ma J, Lin J. Clinical effect of implant denture in prosthodontics. Chinese Community Doctors 2020;36:54-5.

32. Ma L, Yang Z, Liu C. Observation on the application effect of ball cap and Locator attachment implant overdenture in mandibular edentulous jaw restoration. Chinese Journal of Practical Medicine 2019;(19):84-6.

(English Language Editor: J. Jones) 